\title{
Modification research on in wall of capillary copper tube with Norland optical adhesive 68 in a double stereo PCR microfluidic chip
}

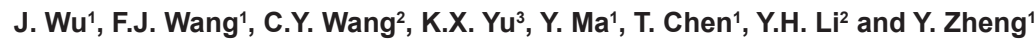 \\ ${ }^{1}$ Institute of Laser Engineering, Beijing University of Technology, Beijing, China \\ ${ }^{2}$ State Key Laboratory of Space Medicine Fundamentals and Application, \\ Chinese Astronaut Science Researching and Training Center, Beijing, China \\ ${ }^{3}$ College of Applied Sciences, Beijing University of Technology, Beijing, China \\ Corresponding author: F. Wang \\ E-mail: yangzheng_12@sohu.com
}

Genet. Mol. Res. 14 (4): 13603-13611 (2015)

Received July 4, 2015

Accepted September 18, 2015

Published October 28, 2015

DOI http://dx.doi.org/10.4238/2015.October.28.21

\begin{abstract}
In this study, a Norland optical adhesive 68 (NOA68) film, approximately $2.2 \mu \mathrm{m}$ thick, was manufactured using ultraviolet solidified NOA68 in apparatus manufacturing film on the inwall of a capillary copper pipe, developed in our laboratory. The roughness of the inwall of capillary copper pipe was improved from $\mathrm{Ra}=0.766$ to $0.204 \mu \mathrm{m}$ and the contact angle was improved from approximately $96^{\circ}$ to $55^{\circ}$, increasing hydrophilicity. Polymerase chain reaction experiments indicated that the ratio of work pressure in the microfluidic chip before modification to that after modification was 2.71/1, indicating that the extension efficiency increased. Our results provide a basis for the construction of a microform chip based on function integration.
\end{abstract}

Key words: Microfluidic chip; Surface modification; NOA68 


\section{INTRODUCTION}

The goal of a miniaturized total analysis system ( $\mu$-TAS) is to integrate all biochemical analysis processes and laboratory functions, such as sampling, dilution, adding reagents, reactions, separation, and detection, on a stamp or a credit card-sized chip at the micro level. This is generally referred to as the $\mu$-TAS "lab on a chip" (Manz et al., 1990; Manz et al., 1993; Shameli et al., 2011). Studies aimed at reducing the working pressure of a microchip will enable reduction of the microchip volume and decrease the requirement for expensive biological reagents. Because the performance of the microchannel inner wall has an important impact on the system working pressure, we altered the working pressure value of a double stereo polymerase chain reaction (PCR) microfluidic chip from a modified microchannel angle and then estimated the reduction in chip volume when pressure decreased.

Currently, PCR microfluidic chips are fabricated by etching or corrosion on a planar substrate to create micro channels, followed by bonding to another substrate (Kopp et al., 1998; Chiou et al., 2001; Nagai et al., 2001; Sun et al., 2002; Obeid and Christopoulos, 2003; Shameli et al., 2011; Iliescu et al., 2012; Fu et al., 2012). During PCR amplification, reagent was injected into the chip and flowed along the microchannel. The microchannel was concurrently divided into 3 temperature regions, and PCR amplification was completed as reagents crossed the chip (Fukuba et al., 2004). Because of the structure of standard microfluidic chips, after PCR reagents flow across the $95^{\circ}, 72^{\circ}$, and $54^{\circ} \mathrm{C}$ temperature zones during the cycle, reagents follow the micro channel across $54^{\circ}, 72^{\circ}$, and $95^{\circ} \mathrm{C}$ to enter the next cycle's $95^{\circ} \mathrm{C}$ temperature zone and begin the next cycle. In this study, a double stereo PCR microfluidic chip composed of a capillary copper tube was used. This allows the PCR reagents to flow from the $54^{\circ} \mathrm{C}$ region directly to the $95^{\circ} \mathrm{C}$ region to being the next cycle, increasing PCR amplification efficiency. Because of the better thermal conductivity of the metal tube in each zone and consistent temperature, $\mathrm{PCR}$ amplification efficiency can be improved. Figure $1 \mathrm{~A}$ shows the structure of a traditional microfluidic chip; Figure $1 \mathrm{~B}$ shows the structure of double stereo PCR microfluidic chip.

In biological experiments, we found that the surface roughness of the microchannel affects the flow of PCR reagents in the microchannel, whereas roughness affects PCR amplification (Yao et al., 2005; Qi et al., 2008; Qi et al., 2009). Because the microchannel wall surface affects fluid flow, the shape and size, surface roughness, and hydrophilicity of the inner wall of the microchannel have an important influence on the performance of the microchannel in fluid mechanics.

Based on centrifugation methods, the surface roughness and hydrophilicity of the capillary copper pipe in the double-dimensional PCR microfluidic chip were altered using Norland optical adhesive NOA 68 to improve the working pressure of the chip and PCR amplification efficiency. This modification method is simple and inexpensive, and most importantly, can modify the relatively closed inwall of the capillary tube, which is difficult using other processes. The products of UV solidifying optical adhesive NOA series has been already used in the related field of biological engineering (Arayanarakool et al., 2010; Dupont et al., 2010; Sollier et al., 2011; Wägli et al., 2011; Mokkapati et al., 2012).

\section{MATERIAL AND METHODS}

\section{Preparation of NOA68 film in capillary copper pipe in wall based on centrifugation}

The capillary copper pipe (external diameter of $1 \mathrm{~mm}$, internal diameter of $0.5 \mathrm{~mm}$ ) used 
in the experiment was made in China. The typical properties of NOA68 are shown in Table 1. The wavelength character of NOA68 is shown in Figure 2.

A

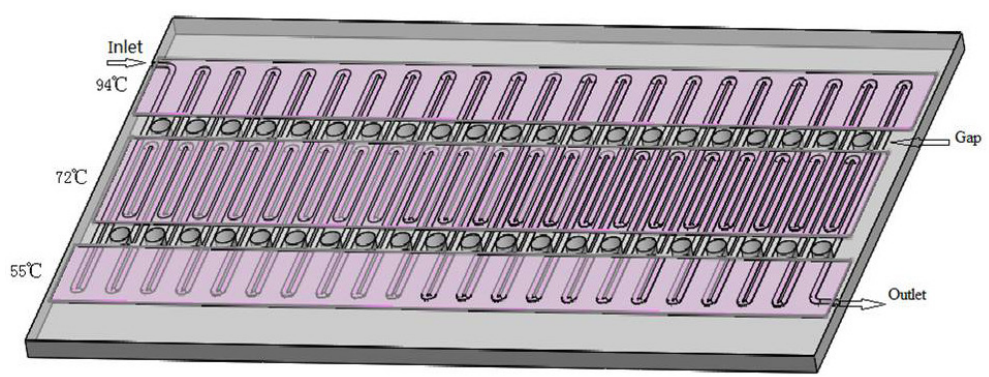

B

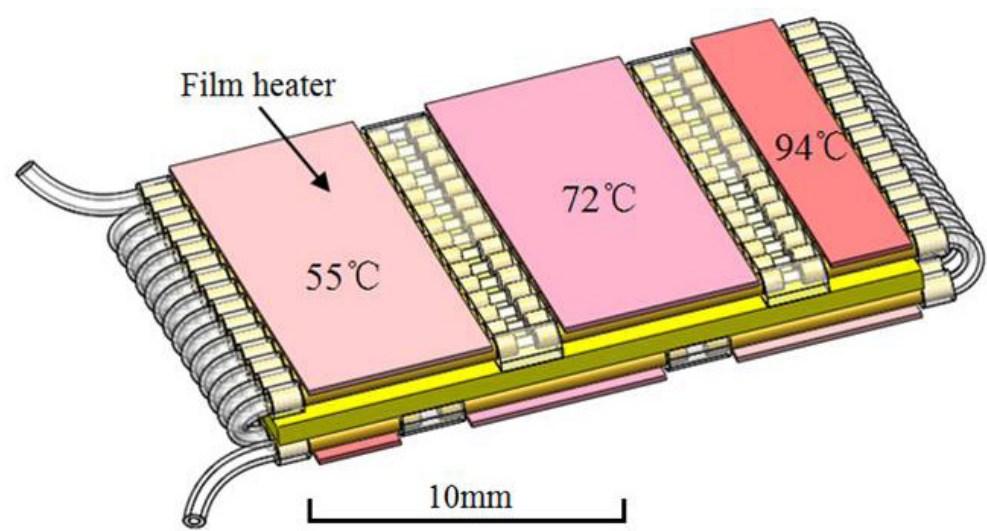

Figure 1. Structure of 2 types of microfluidic chips.

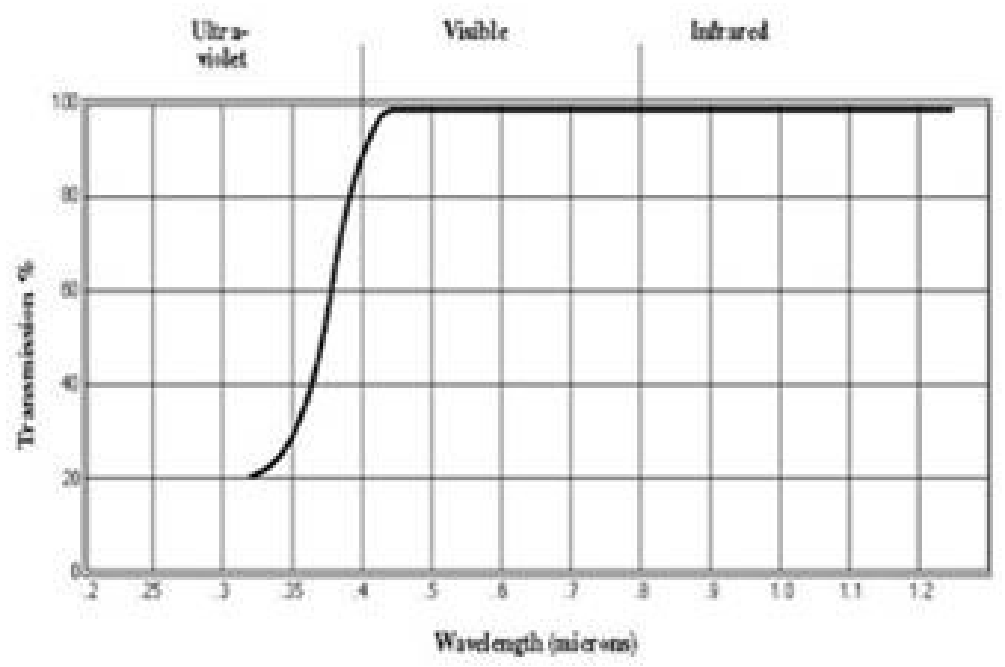

Figure 2. Wavelength character of NOA68. 
Table 1. Typical properties of NOA68.

\begin{tabular}{lc}
\hline Properties & \\
\hline Solids & $100 \%$ \\
Viscosity at $25^{\circ} \mathrm{C}$ & $5000 \mathrm{cps}$ \\
Refractive Index of Cured Polymer & 1.54 \\
Elongation at Failure & $80 \%$ \\
Modulus of Elasticity (psi) & 20,000 \\
Tensile Strength (psi) & 2,500 \\
Hardness - Shore D & 60 \\
\hline
\end{tabular}

\section{Preparation of NOA68 film in capillary copper pipe}

First, NOA68 was injected into the capillary copper pipe, which had been cut to an appropriate size. The capillary copper pipe was rotated along the Y-axis at $120 \mathrm{r} / \mathrm{s}$ to draw out excess NOA68. Next, the pipe was rotated along the X-axis at $0.5 \mathrm{r} / \mathrm{s}$ during exposure to a $355 \mathrm{~nm}$ laser with diameter of $3 \mu \mathrm{m}$ to scan around the pipe and procure the NOA68 inside (Figure 2 and $3)$. Finally, the preprocessed capillary copper pipe was placed under a high-power mercury lamp (working wavelength of $365 \mathrm{~nm}$ ) for $15 \mathrm{~min}$ until the NOA68 was fully cured. During curing, the tubular axis and curing axis were coaxial.

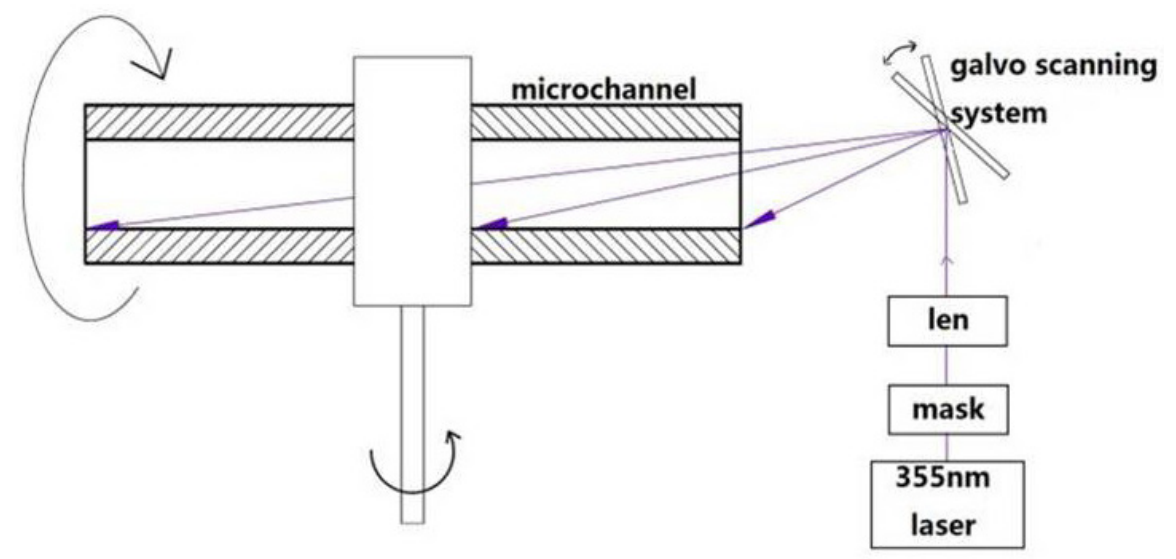

Figure 3. Sketch map of the preparation of NOA68 film in capillary copper pipe.

Capillary copper tube and elbow of quartz material assemble into a double microfluidic chip. NOA68 is injected and filled of microchannel chip, then with compressed air into the entrance of the chip,NOA68 which is in microchannels would be extruded from the outlet of the chip, while UV light (High-power lamp, wave length $365 \mathrm{~nm}$ ) through the quartz material elbow into the capillary brass at the front and back of the chip, make axis of capillary copper tube and UV light coaxial (Figure 4), NOA68 could be complete consolidated after continuous radiation of 15 minutes. Then modification on inwall of capillary copper tube with NOA68 in double stereo PCR microfluidic chip is achieved.

\section{Characterization of inner wall films of copper capillary using centrifugation}

The copper capillary was cut axially before modification and we measured the roughness using a confocal microscope (LEXT-OLS3100, Olympus, Tokyo, Japan). 


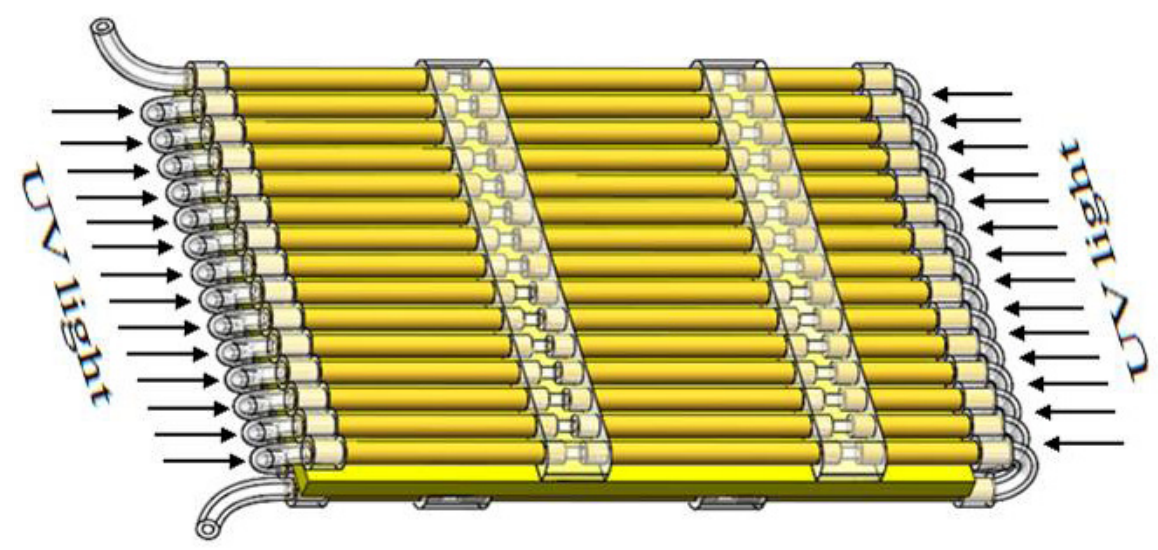

Figure 4. Modification on in wall of capillary copper tube with NOA68 in double stereo PCR microfluidic chips.

Because the inner wall of the copper capillary is semi-circular, its contact angle cannot be directly measured. In this study, the samples of copper plate used were provided by copper capillary manufacturers, so the copper plate and copper capillary were composed of the same material. The copper plate samples were polished so that it had an equivalent roughness to the copper capillary. The roughness of both samples was measured using the same instrument. The thin film was manufactured by using NOA68 in a portion of the area of the copper plate. The contact angle was measured using an optical meter (OCA20, Data Physics Corp., San Jose, CA, USA) and NOA68 film thickness was measured using a 3D profiler (Wyko NT1100, Veeco, Plainview, NY, USA).

\section{Comparison of working pressure of chip inside system and PCR amplification efficiency}

The experimental conditions for the comparison of chips were as follows: 1) the same physical parameters (such as temperature, sample injection rate, etc.) and fluorescent PCR reagent; 2) equal experimental time and same circulating times of PCR for the PCR amplification in the chip comparison; and 3) the same physical parameters (such as detector operating voltage, etc.) for the fluorescence detection instrument.

Comparison of microfluidic control pressure on the chips using the fluorescent PCR reagent was accomplished under the conditions described above. Then, the working pressure values for the inside system of the PCR microfluidic chip were obtained after these modification.

The original signal values of the fluorescence PCR reagents were detected using a fluorescence detection instrument (excitation wavelength: $475 \mathrm{~nm}$, detection wavelength: $530 \mathrm{~nm}$ ). The original signal values of the fluorescence PCR reagents refers to the numbers without units obtained from the optical system when excitation units and detection units were both operating in the fluorescence detection system. The data included the original signal values, which were not amplified by the digital processing circuit. This directly reflects the resolution or sensitivity of the fluorescence signal in fluorescence detection system experiments (Figures 5 and 6). To stabilize the changing amplification temperature field of the chips and reduce the influence of external light on the fluorescence intensity, a black neutral cure silicone sealant, Silicone construction sealant TOSSEAL 381 (Akasaka Minato-ku, Tokyo JAPAN), was used to package the chipset. In Figure 
7, 1 refers to the PCR microfluidic chipset with a double-layer stereoscopic copper capillary of 36 PCR amplification cycles, 2 refers to the PCR reagent injection system, and 3 refers to the working pressure monitoring system.

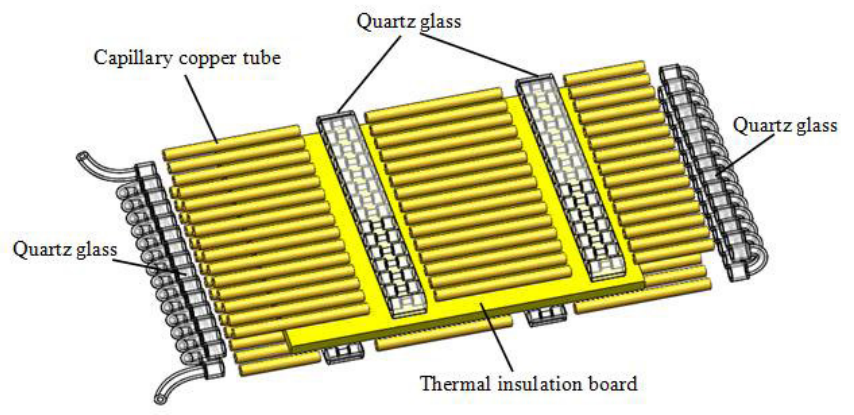

Figure 5. Decomposition structure diagram of double-layer stereoscopic comparison PCR microfluidic chips.

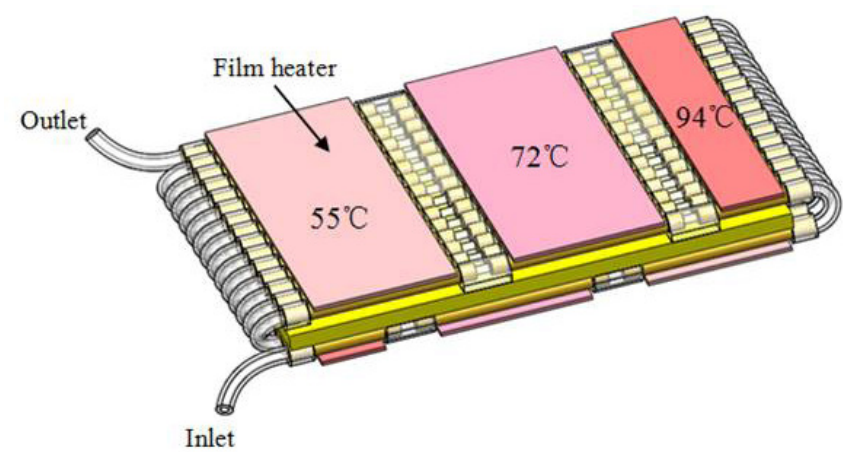

Figure 6. Whole structure diagram of the double-layer stereoscopic comparison PCR microfluidic chips.

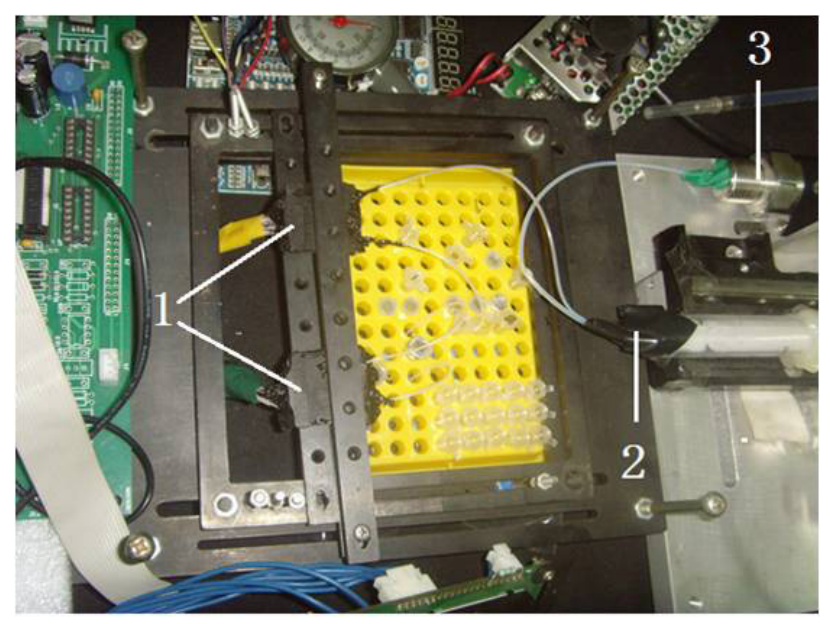

Figure 7. Experiment comparing the chips. 


\section{RESULTS}

\section{Manufacture of film NOA68 in capillary copper pipe based on centrifugation}

The roughness of the inwall of the capillary copper pipe was improved from $\mathrm{Ra}=0.766$ to $0.204 \mu \mathrm{m}$ (Figure 8). A smaller contact angle resulted in stronger hydrophilicity. The film NOA68 improved the hydrophilicity of the inwall of the capillary copper pipe, as shown in Figure 9. The red line indicates the value before modification, while the black line is the result after modification. The thickness of the film NOA68 formed on the copper plate was $2.2 \mu \mathrm{m}$.
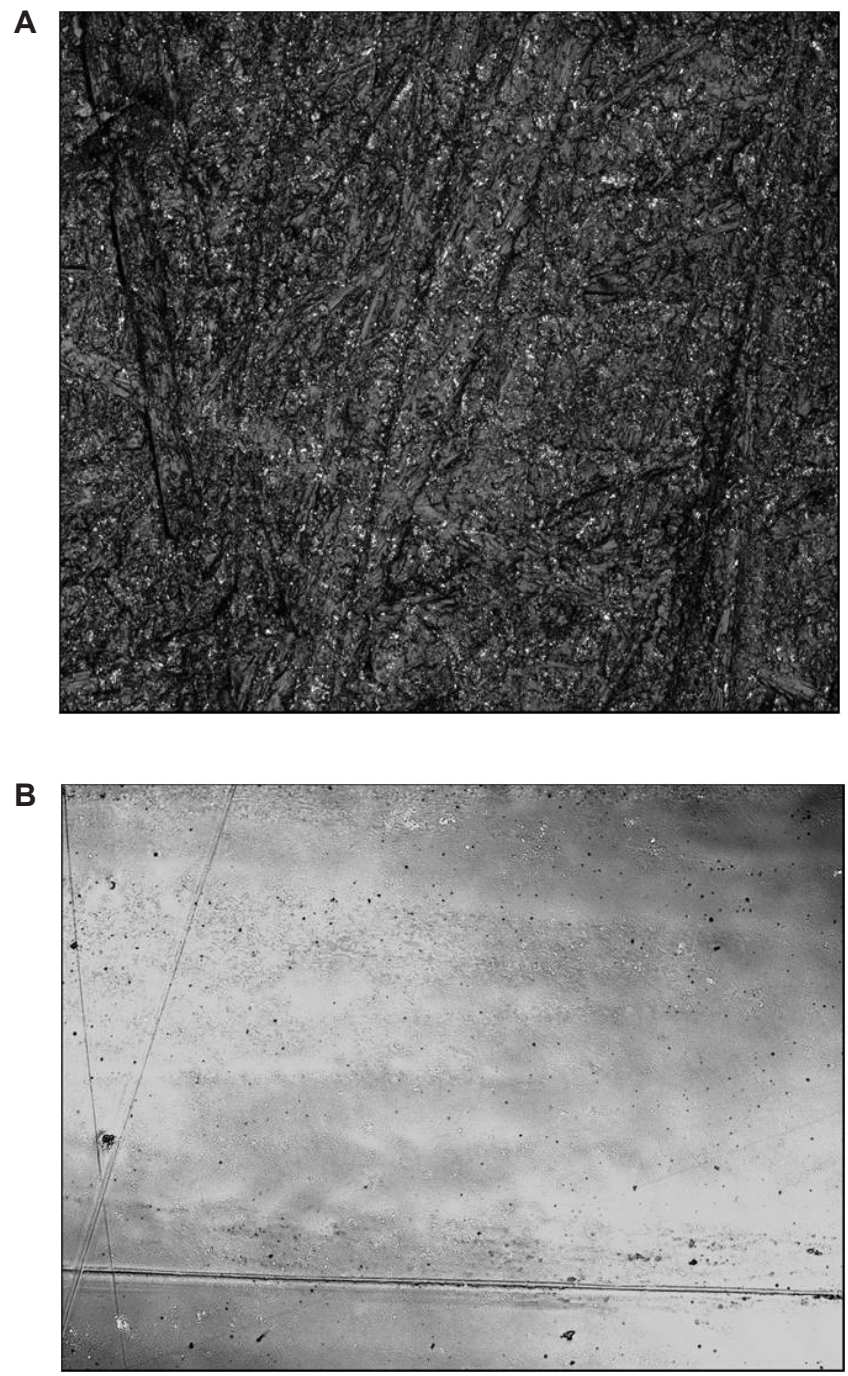

Figure 8. Inside wall of capillary copper pipe. 


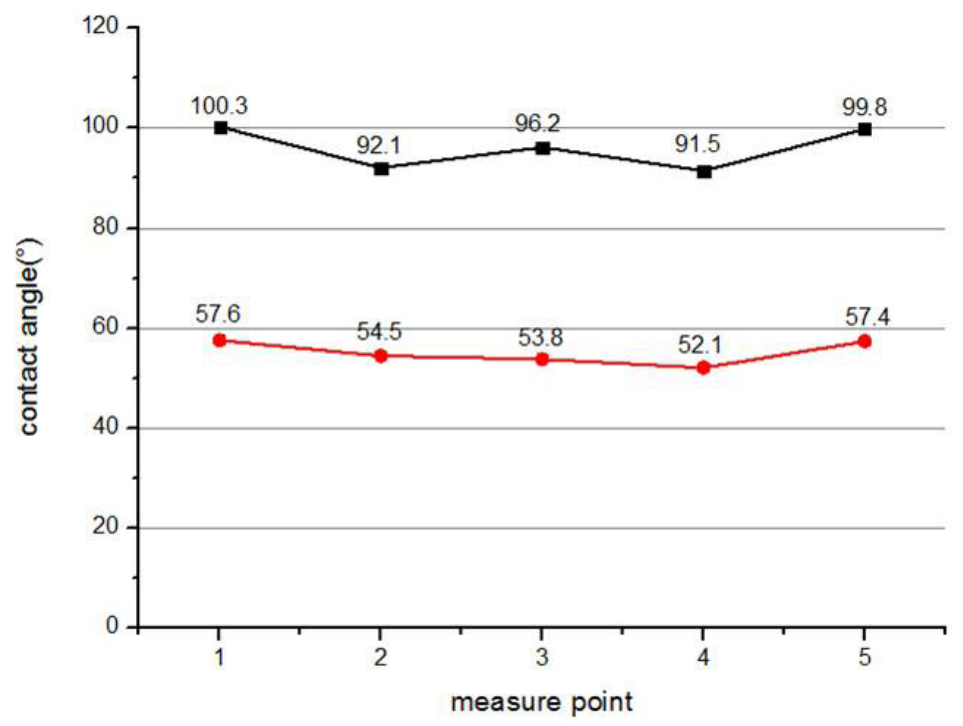

Figure 9. Measurement results of contact angle of copper plate.

\section{Contrast result and analysis of system work pressure in chip and extension efficiency of PCR}

For contrasting system work pressure in the chip and the extension efficiency of PCR, 36 PCR extension circle analogical experiments were conducted using pure water. The ratio of the system work pressure in the microfluidic PCR chip before and after roughness modification was 2.71/1 (average value). The fluorescence original value before and after extension of PCR is shown in Table 2. This value indicates that improving the roughness can increase the extension efficiency of PCR in the chip. The fluorescence original value of PCR was higher after extension than before extension. The reason is that the improving roughness can reduce "hang wall" phenomenon of big molecule.

Table 2. Fluorescence original value before and after extension of PCR.

\begin{tabular}{lcc}
\hline & Before extension of PCR & After extension of PCR \\
\hline Before modifying & 101 & 456 \\
After modifying & 103 & 712 \\
\hline
\end{tabular}

\section{DISCUSSION}

The film NOA68 was manufactured in the inwall of the capillary copper pipe of a doublelayer and 3-dimensional microfluidic PCR chip on using centrifugation. The ratio of the system work pressure in the microfluidic PCR chip before and after roughness modification was 2.71/1, increasing PCR extension efficiency.

It is assessed that this treatment may allow for a 1.5-fold reduction in the chip dimension. Our results provide a basis for a construction microform of the chip based on function integration. This microfabrication based on functional integration provides the basis for manufacturing an alarm 
system for detecting microbial growth in clinical diagnosis and space research.

\section{Conflicts of Interest}

The authors declare no conflict of interest.

\section{ACKNOWLEDGMENTS}

Research supported by the National Natural Science Foundation of China (Grant \#51375024), Scientific-Technical Project ofBeijing Education Committee(Grant\#KZ201210005009), and the Opening Foundation of the State Key Laboratory of Space Medicine Fundamentals and Application, Chinese Astronaut Research and Training Center (Grant \#SMFA13K06).

\section{REFERENCES}

Arayanarakool R, Le Gac S and van den Berg A (2010). Low-temperature, simple and fast integration technique of microfluidic chips by using a UV-curable adhesive. Lab. Chip 10: 2115-2121.

Chiou J, Matsudaira P, Sonin A and Ehlrich D (2001). A closed-cycle capillary polymerase chain reaction machine. Anal. Chem. 73: 2018-2021.

Dupont EP, Luisier R and Gijs MAM (2010). NOA 63 as a UV-curable material for fabrication of microfluidic channels with native hydrophilicity. Microelectron. Eng. 87: 1253-1255.

Fu LM, Ju WJ, Yang RJ and Wang YN (2012). Rapid prototyping of glass-based microfluidic chips utilizing two-pass defocused $\mathrm{CO}_{2}$ laser beam method. Microfluid. Nanofluid. 14: 479-487.

Fukuba T, Yamamoto T, Naganuma T and Fujii T (2004). Microfabricated flow-through devices for DNA ampification - towards in situ gene analysis. Chem. Eng. J. 101: 151-156.

Iliescu C, Taylor H, Avram M, Miao J, et al. (2012). A practical guide for the fabrication of microfluidic devices using glass and silicon. Biomicrofluidics 6: 16505-1650516.

Kopp MU, Mello AJ and Manz A (1998). Chemical amplification: continuous-flow PCR on a chip. Science 280: 1046-1048.

Manz A, Graber N and Widmer HM (1990). Miniaturized total chemical-analysis systems-a novel concept for chemcal sensing. Sensor Actuat. B Chem. 1: 244-248.

Manz A, Harrison DJ and Verpoorte E (1993). Planar chip technology of separation system: a developing perspective in chemical monitoring. In: Advances in Chromatography. Vol. 33. Marcel Dekker, New York, 1-66.

Mokkapati VRSS, Bethge $O$ and Hainberger R (2012). Microfluidic chips fabrication from UV curable adhesives for heterogeneous integration. 62nd IEEE Electronic Components Technology Conference (ECTC) 8: 1965-1969.

Nagai H, Murakami Y, Morita Y, Yokoyama K, et al. (2001). Development of a microchamber array for picoliter PCR. Anal. Chem. 73: 1043-1047.

Obeid PJ and Christopoulos TK (2003). Continuous-flow DNA and RNA amplification chip combined with laser-induced fluorescence detection. Anal. Chim. Acta 494: 1-9.

Qi H, Chen T, Yao L and Zuo T (2008). Hydrophilicity modification of poly(methyl methacrylate) by excimer laser ablation and irradiation. Microfluid. Nanofluid. 5: 139-143.

Qi H, Wang XS, Chen T, Ma XN, et al. (2009). Fabrication and characterization of a polymethyl methacrylate continuous-flow PCR microfluidic chip using CO2 laser ablation. Microsyst. Technol. 15: 1027-1030.

Shameli SM, Elbuken C, Ou J, Ren CL, et al. (2011). Fully integrated PDMS/SU-8/quartz microfluidic chip with a novel macroporous poly dimethylsiloxane (PDMS) membrane for isoelectric focusing of proteins using whole-channel imaging detection. Electrophoresis 32: 333-339.

Sollier E, Murray C, Maoddi P and Di Carlo D (2011). Rapid prototyping polymers for microfluidic devices and high pressure injections. Lab Chip 11: 3752-3765.

Sun K, Yamaguchi A, Ishida Y, Matsuo S, et al. (2002). A heater-integrated transparent microchannel chip for continuous-flow PCR. Sensor. Actuat. B Chem. 84: 283-289

Wägli P, Homsy A and de Rooij NF (2011). Norland optical adhesive (NOA81) microchannels with adjustable wetting behavior and high chemical resistance against a range of mid-infared-transparent organic solvents. Sensor. Actuat. B Chem. 156: 994-1001.

Yao L, Liu B, Chen T, Liu S, et al. (2005). Micro flow-through PCR in a PMMA chip fabricated by KrF excimer laser. Biomed. Microdevices 7: 253-257. 